\title{
Utilização da oxigenoterapia hiperbárica no tratamento da doença vascular periférica: uma revisão sistemática
}

\author{
Use of hyperbaric oxygen therapy in the treatment of peripheral vascular disease: \\ systematic review
}
Uso de la oxigenoterapia hiperbárica en el tratamiento de la enfermedad vascular periférica: revisión sistemática

Erika de Oliveira Menezes ${ }^{1 \star}$, Bruno Barreto Cintra ${ }^{1}$, Victor Hugo Cardoso Félix¹.

\section{RESUMO}

Objetivo: Avaliar o conhecimento e eficácia da oxigenoterapia hiperbárica em pacientes com doença vascular periférica, além de elucidar seus efeitos colaterais e fisiológicos nestes pacientes. Métodos: Trata-se de uma revisão sistemática integrativa com pesquisa bibliográfica através das seguintes bases de dados: U.S. National Library of Medicine National Institute of Health (PubMed), Scientific Electronic Library Online (SCIELO) e Literatura Latino-Americana e do Caribe em Ciências da Saúde (LILACS). Os descritores utilizados foram "Peripheral Arterial Disease AND Hyperbaric Oxygenation", Hyperbaric Oxygenation AND Wound" e "feridas complexas AND oxigenoterapia hiperbárica", respectivamente. Resultados: Foram incluídos 15 artigos nesta revisão, 10 publicados em inglês, 4 em português e 1 em espanhol. Os artigos abordaram a eficácia da oxigenoterapia hiperbárica como terapia adjuvante de lesões causadas por doença arterial periférica e de outras feridas complexas. Considerações finais: A oxigenoterapia hiperbárica apresenta boa eficácia no tratamento adjuvante de feridas complexas, incluindo as lesões por doença arterial periférica, úlceras em pés diabéticos, além das feridas agudas relacionadas a trauma.

Palavras-chave: Doença arterial periférica, Oxigenoterapia, Oxigenação hiperbárica.

\begin{abstract}
Objective: To evaluate the knowledge and effectiveness of hyperbaric oxygen therapy in patients with peripheral vascular disease, in addition to elucidating the side and physiological effects of hyperbaric oxygen therapy in these patients. Methods: This is a systematic integrative review with bibliographic research through the following databases: US National Library of Medicine National Institute of Health (PubMed), Scientific Electronic Library Online (SCIELO) and Latin American and Caribbean Literature in Health Sciences (LILACS). The descriptors used were "Peripheral Arterial Disease AND Hyperbaric Oxygenation", "Hyperbaric Oxygenation AND Wound" and "Complex wounds AND hyperbaric oxygen therapy", respectively. Results: 15 articles were included in this review, 10 published in English, 4 in Portuguese and 1 in Spanish. The articles addressed the efficacy of hyperbaric oxygen therapy as an adjunct therapy to injuries caused by peripheral arterial disease and other complex wounds. Conclusion: Hyperbaric oxygen therapy has good efficacy in the adjuvant treatment of complex wounds, including injuries due to peripheral arterial disease, ulcers in diabetic feet, in addition to acute trauma-related wounds.
\end{abstract}

Key words: Peripheral arterial disease, Oxygen therapy, Hyperbaric oxygenation.

1 Universidade Tiradentes (UNIT), Aracaju - SE. *E-mail: erikaomenezess@gmail.com

SUBMETIDO EM: 9/2020

ACEITO EM: 10/2020

PUBLICADO EM: 11/2020

REAS/EJCH | Vol.12(11) | e5282 | DOI: https://doi.org/10.25248/reas.e5282.2020 Página 1 de 11 


\section{RESUMEN}

Objetivo: Evaluar el conocimiento y la eficacia de la oxigenoterapia hiperbárica en pacientes con enfermedad vascular periférica, además de dilucidar sus efectos secundarios y fisiológicos en estos pacientes. Métodos: Esta es una revisión integradora sistemática con investigación bibliográfica a través de las siguientes bases de datos: Biblioteca Nacional de Medicina de EE. UU. Instituto Nacional de Salud (PubMed), Biblioteca Electrónica Científica en Línea (SCIELO) y Literatura Latinoamericana y del Caribe en Ciencias de la Salud. (LILACS). Los descriptores utilizados fueron "Enfermedad Arterial Periférica Y Oxigenación Hiperbárica", Oxigenación Hiperbárica $Y$ Herida" y "Heridas complejas $Y$ oxigenoterapia hiperbárica", respectivamente. Resultados: En esta revisión se incluyeron 15 artículos, 10 publicados en inglés, 4 en portugués y 1 en español. Los artículos abordaron la eficacia de la oxigenoterapia hiperbárica como terapia complementaria para las lesiones causadas por la enfermedad arterial periférica y otras heridas complejas. Conclusión: La oxigenoterapia hiperbárica tiene una buena eficacia en el tratamiento adyuvante de heridas complejas, incluidas las debidas a arteriopatía periférica, úlceras en los pies diabéticos, además de heridas agudas relacionadas con traumatismos.

Palabras clave: Enfermedad arterial periférica, Oxigenoterapia, Oxigenación hiperbárica.

\section{INTRODUÇÃO}

O termo doença vascular periférica ou doença arterial periférica (DAP) designa a estenose ou oclusão nas artérias das extremidades inferiores, que se mostram, na maioria das vezes, assintomáticas. Os pacientes sintomáticos podem apresentar desde sintomas leves, como claudicação ao esforço físico, até sintomas graves como úlceras complexas, decorrentes, principalmente, da aterosclerose, ocorrendo em repouso com isquemia contínua dos membros. A incidência da doença vascular periférica em adultos jovens é desprezível, mas está presente em $10 \%$ a $30 \%$ dos adultos com mais de 50 anos, sendo a DAP um marcador de presença da doença coronária e de doença vascular cerebral (COSTA RV, et al., 2017). Acidentes vasculares cerebrais e infartos do miocárdio ocorrem numa frequência 3 vezes maior que naqueles sem a doença.

O principal processo patológico que pode causar obstrução arterial, levando a sintomas de insuficiência arterial pela redução do fluxo sanguíneo, é a aterosclerose, que começa pela lesão ao endotélio vascular e a formação de uma placa aterosclerótica. Esse processo é potencializado pela presença de inflamação local mediada por leucócitos e por oxidação de lipoproteínas. Alguns fatores de risco da doença são a idade acima de 70 anos, tabagismo, diabetes mellitus, hipercolesterolemia e hipertensão arterial sistêmica, mas o mecanismo de ação destes na fisiopatologia da doença ainda não foi bem elucidado (CONTE M, et al., 2019).

As manifestações clínicas de claudicação, dor e parestesia em membros inferiores, além de úlceras isquêmicas, são beneficiadas de terapia clínica e da revascularização cirúrgica do vaso aterosclerótico. No entanto, a mortalidade operatória varia de 1 a $3 \%$, fazendo com que os benefícios da cirurgia sejam cuidadosamente pesados e novas terapias surjam, inclusive como adjuvantes, no tratamento das úlceras e lesões gangrenosas, como a oxigenoterapia hiperbárica (FAUCI AS, et al., 2006).

O oxigênio é indispensável para a atividade dos polimorfonucleares, para a ativação dos fibroblastos e para a hidroxilação dos aminoácidos lisina e prolina, que é uma etapa fundamental dessa síntese. O estímulo básico para a angiogênese é a hipóxia. A oxigenoterapia hiperbárica $(\mathrm{OHB})$ provoca um aumento no gradiente de pressão de oxigênio entre os tecidos normais e os lesados, potencializando, portanto, o estímulo para seu desencadeamento. Em feridas epiteliais, a OHB aumentou a resposta angiogênica, demonstrada por análises histológicas, e, além disso, um aumento da vascularização claramente foi notado (NAKAMURA $H$, et al., 2020).

A oxigenoterapia hiperbárica (OHB) constitui-se da inalação intermitente de oxigênio a $100 \%$ sob uma pressão atmosférica superior a $1 \mathrm{~atm}$, o que determina um aumento nos níveis de oxigênio dissolvidos no plasma sanguíneo. A OHB é comumente usada em condições de hipóxia ou para tratar embolia gasosa, na qual bolhas de gás obstruem o fluxo sanguíneo (COSTA RV, et al., 2017). 
Muitos dos seus efeitos são mediados por espécies reativas de oxigênio que são gerados pelo próprio tecido, induzidos pela hiperóxia. Com o aumento da pressão atmosférica, em um ambiente fechado, as câmaras, os pacientes entram em um estado de hiperóxia, onde a concentração de oxigênio dissolvido no plasma aumenta de $0,3 \mathrm{ml} / \mathrm{dl}$ para $6,0 \mathrm{ml} / \mathrm{dl}$. Essa concentração é suficiente para atender aos requisitos de extração de oxigênio do tecido em repouso, independente da adequação do ligamento entre oxigênio e hemoglobina, o que fez a oxigenoterapia hiperbárica ser utilizada em condições de isquemia e de envenenamento por monóxido de carbono, por exemplo (NAKAMURA H, et al., 2020).

O estado de hiperóxia só é conseguido pelo uso de câmaras hiperbáricas, que permitem o isolamento do ambiente, capazes de acomodar um, se o paciente for crítico, ou mais pacientes, em casos de terapia de condições clínicas crônicas. Tratamentos agudos geralmente demandam uma ou duas sessões de oxigenoterapia, enquanto o tratamento de doenças crônicas, como as úlceras por doença vascular periférica, precisa de até 30 sessões. Caso a pressão no ambiente exceda $3 \mathrm{~atm}$, há um risco dramático de neurotoxicidade pelo estado de alta exposição ao oxigênio. No entanto, sua única contraindicação absoluta documentada é o pneumotórax não tratado (MECHEM CO, et al., 2020).

No tratamento de feridas complexas crônicas, como as lesões da doença arterial periférica, a oxigenoterapia hiperbárica é usada como adjuvante, preparando o leito da ferida, aumentando a perfusão local de oxigênio no tecido antes do procedimento cirúrgico definitivo (ANDRADE SVR, et al., 2016). Além desses benefícios, segundo Kimmel HM, et al. (2016), a oxigenoterapia hiperbárica também promove a redução do edema, a queda na regulação das citocinas inflamatórias, a proliferação de fibroblastos, produção de colágeno e angiogênese. No entanto, ainda são escassas as pesquisas sobre esse tema, principalmente no Brasil.

Dessa forma, diante da magnitude epidemiológica da doença vascular periférica na nossa população, assim como do pequeno número de estudos relacionados a oxigenoterapia hiperbárica, propõe-se, com essa revisão, avaliar a eficácia da medicina hiperbárica nessa população, bem como elucidar o conhecimento sobre os efeitos fisiológicos e colaterais da terapia nesses pacientes.

\section{MÉTODOS}

Refere-se a uma revisão sistemática com levantamento bibliográfico realizado através de estudos primários conduzidos com o objetivo de entender mais sobre a utilização da oxigenoterapia hiperbárica no tratamento de feridas complexas, principalmente em portadores de doença arterial periférica. O levantamento sistematizado ocorreu entre os meses de agosto e setembro de 2020.

A revisão foi feita seguindo os moldes de uma revisão integrativa, aprofundando-se a partir dos seguintes passos: definição da questão de pesquisa; definição dos critérios de elegibilidade; identificação e seleção dos artigos; categorização dos estudos selecionados; avaliação da elegibilidade dos estudos; análise e interpretação dos resultados com a síntese dos dados e apresentação dos resultados finais.

A pesquisa bibliográfica foi realizada com as bases de dados U.S. National Library of Medicine National Institute of Health (PubMed), Scientific Electronic Library Online (SCIELO) e Literatura Latino-Americana e do Caribe em Ciências da Saúde (LILACS), com os seguintes descritores: "Peripheral Arterial Disease AND Hyperbaric Oxygenation" e "Hyperbaric Oxygenation AND Wound", além de "feridas complexas AND oxigenoterapia hiperbárica”, respectivamente.

Para selecionar os artigos de interesse, foi realizada uma leitura prévia do título e resumo, onde foram selecionados os textos condizentes com a temática desta revisão. Em seguida, os artigos foram filtrados de acordo com os critérios de inclusão e exclusão definidos anteriormente, a partir da leitura seletiva dos resumos. E, por fim, ocorreu a etapa de extração de dados, que permitiu analisar minuciosamente os artigos na íntegra, e selecionar os estudos relevantes para a revisão.

Foram incluídos neste estudo, artigos publicados em inglês, espanhol e português entre 2012 e 2020, por se tratar de um período atualizado, além de artigos que tinham como tema principal o uso de oxigenoterapia 
hiperbárica no tratamento de feridas complexas. Os artigos excluídos foram os publicados antes de 2012 e aqueles que não tiveram como tema o proposto pela pesquisa.

A pesquisa foi feita através de dados disponibilizados eletronicamente e, por ser uma revisão sistemática, não foi necessária a aprovação do Comitê de Ética em Pesquisa (CEP). Após realização da busca, os artigos foram exportados e agrupados no gerenciador de referências Zotero para análise e exclusão de duplicatas de forma automatizada.

\section{RESULTADOS}

Em um primeiro momento, a busca dos artigos se deu na base de dados PUBMED, na qual os descritores utilizados foram "Peripheral Arterial Disease AND Hyperbaric Oxygenation", resultando em 26 artigos. Para afunilar a pesquisa, os filtros utilizados foram: texto completo gratuito e entre 2012 e 2020; o que resultou em 6 artigos.

Em um segundo momento, a pesquisa foi realizada na plataforma SCIELO com os seguintes descritores: "Hyperbaric Oxygenation AND Wound", o que resultou em 75 artigos. A partir do filtro de publicação entre 2012 e 2020, restaram 5 artigos com o tema de oxigenoterapia hiperbárica no tratamento de feridas.

$\mathrm{Na}$ base de dados LILACS, foram utilizados os descritores "feridas complexas AND oxigenoterapia hiperbárica", resultando em 21 artigos. Destes, 17 artigos foram excluídos por não se adequarem aos critérios de inclusão, restando apenas 4 artigos. Vale destacar, então, que esta revisão integrativa avaliou um total de 15 artigos, como demonstrado na Figura 1.

Figura 1 - Fluxograma demonstrando as etapas de seleção da revisão.

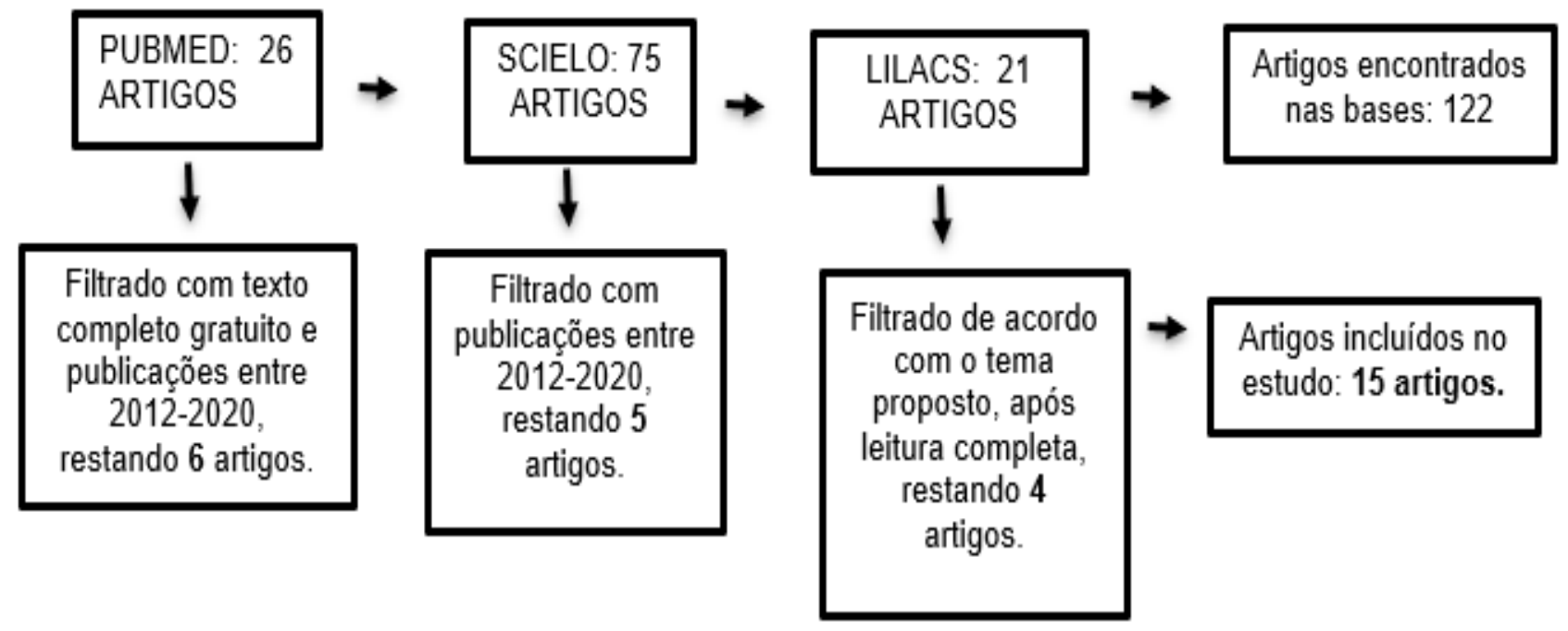

Fonte: Menezes EO, et al., 2020.

Após seleção dos artigos, foram observados os seguintes aspectos: amostra, objetivos e resultados, além de ano de publicação, como demonstra a Tabela 1. Dos 15 artigos selecionados para compor esta revisão, 10 foram publicados em inglês, 1 em espanhol e 4 em português.

Destes 15 artigos, 4 foram publicados em 2013, 3 foram publicados em 2016 e 2 em 2018, sendo os anos com maior quantidade de publicações. Em relação a metodologia usada nos estudos, $53,4 \%$ (8 artigos) dos estudos são transversais, $13,4 \%$ (2) são relatos de caso, 6,7\% (1) são revisões de literatura; os demais utilizaram delineamento de casos e controles. Dentre os artigos que utilizaram pacientes ou prontuários, todos apresentaram pacientes entre 19 e 89 anos. Nenhum estudo incluiu crianças. Todos os trabalhos mencionaram as questões de natureza ética. 
Tabela 1 - Artigos incluídos na revisão sistemática.

\begin{tabular}{|c|c|c|c|}
\hline Autor & Ano de publicação & Objetivos & Resultados \\
\hline Adorno ETF, et al. & 2013 & $\begin{array}{l}\text { Demostrar o perfil epidemiológico dos } \\
\text { pacientes tratados com auxílio da } \\
\text { oxigenioterapia hiperbárica no Estado } \\
\text { de Mato Grosso do Sul, de maio } 2007 \\
\text { a outubro } 2012 \text {. }\end{array}$ & $\begin{array}{l}\text { A cirurgia plástica foi a especialidade que mais solicitou OHB } \\
\text { associada ao tratamento convencional de seus pacientes } \\
(71 \% \text { do total de pacientes), sendo destes a maioria devido à } \\
\text { lesão por queimadura ( } 82 \%) \text {. Nas demais especialidades, a } \\
\text { cirurgia vascular responde por } 17 \% \text { dos pacientes. }\end{array}$ \\
\hline Andrade SM, Santos ICRV. & 2016 & $\begin{array}{l}\text { Descrever os tipos mais frequentes de } \\
\text { feridas com indicação para terapia por } \\
\text { oxigênio hiperbárico e os resultados } \\
\text { obtidos. }\end{array}$ & $\begin{array}{l}\text { As feridas mais frequentemente encontradas como indicação } \\
\text { para terapia por oxigênio hiperbárico foram: úlcera venosa, } \\
\text { lesão traumática e pé diabético. Os pacientes com feridas } \\
\text { crônicas realizaram um menor número de sessões }(61,1 \%) \text { e } \\
\text { tiveram suas feridas cicatrizadas ou reduzidas }(62,0 \%) \\
\text { quando comparados com aqueles com feridas agudas. }\end{array}$ \\
\hline Araujo FM, et al. & 2013 & Relato de caso & $\begin{array}{l}\text { Apresentação do caso de um paciente masculino, } 50 \text { anos, } \\
\text { com úlcera extensa e profunda em perna esquerda, que } \\
\text { exemplifica bem a dificuldade terapêutica e que evoluiu com } \\
\text { excelente resultado após associação de oxigenoterapia } \\
\text { hiperbárica e enxertia de pele ao tratamento imunossupressor } \\
\text { inicialmente proposto. }\end{array}$ \\
\hline Berner JE, et al. & 2014 & $\begin{array}{l}\text { O artigo é uma revisão de literatura que } \\
\text { buscou mostrar as bases físicas e } \\
\text { biológicas para o uso da oxigenoterapia } \\
\text { hiperbárica. }\end{array}$ & $\begin{array}{l}\text { A difusão das aplicações da oxigenoterapia hiperbárica no } \\
\text { tratamento de feridas pode favorecer a instalação de novas } \\
\text { câmeras e a formação de mais especialistas. Dessa forma, } \\
\text { não só poderia ser melhorado o acesso aos pacientes com } \\
\text { feridas em cicatrização, mas também facilitaria novas } \\
\text { pesquisas sobre seu uso. }\end{array}$ \\
\hline Columbo JA, et al. & 2016 & $\begin{array}{l}\text { Relato de } 4 \text { casos com pacientes pós } \\
\text { amputação por doença arterial } \\
\text { periférica, cujas feridas nos cotos não } \\
\text { cicatrizavam até serem submetidos a } \\
\text { oxigenoterapia periférica. }\end{array}$ & $\begin{array}{l}\text { Com a adição do tratamento com oxigênio hiperbárico ao } \\
\text { cuidado vigilante da ferida e à terapia de pressão negativa, } \\
\text { esses pacientes curaram seus cotos de amputação e } \\
\text { receberam próteses. No acompanhamento mais recente, } \\
\text { todos os pacientes estavam deambulando e usando suas } \\
\text { próteses. }\end{array}$ \\
\hline
\end{tabular}

$\overline{\text { REAS/EJCH | Vol.12(11) | e5282 | DOI: https://doi.org/10.25248/reas.e5282.2020 Página } 5 \text { de } 11}$ 


\begin{tabular}{|c|c|c|c|}
\hline Autor & Ano de publicação & Objetivos & Resultados \\
\hline Dünnwald T, et al. & 2018 & $\begin{array}{l}\text { Avaliar se a combinação da } \\
\text { oxigenoterapia hiperbárica com o } \\
\text { aquecimento de corpo inteiro usando a } \\
\text { tecnologia infravermelha de baixa } \\
\text { temperatura (HBOIR) pode melhorar as } \\
\text { lesões por hipoxemia. }\end{array}$ & $\begin{array}{l}\text { Tanto a tecnologia infravermelha de baixas temperaturas } \\
\text { quanto a oxigenoterapia hiperbárica (HBO) aumentam a } \\
\text { distância de difusão do oxigênio nos tecidos. No entanto, as } \\
\text { respostas fisiológicas e a segurança de oxigenoterapia } \\
\text { hiperbárica com radiação infravermelha (HBOIR), em um } \\
\text { aumento de oxigênio pressão parcial de } 1,4 \text { bar ou } 2,4 \\
\text { atmosferas absolutas (ATA) ainda precisa ser determinada. A } \\
\text { esperança é que apareçam estudos futuros para examinar sua } \\
\text { eficácia em pacientes com condições clínicas, que incluem } \\
\text { doença arterial periférica (DAP) ou distúrbios de cicatrização } \\
\text { de feridas. }\end{array}$ \\
\hline Duran EP, et al. & 2012 & $\begin{array}{l}\text { Avaliar o uso de oxigenoterapia } \\
\text { hiperbárica no tratamento de feridas } \\
\text { por doença arterial obstrutiva crônica. }\end{array}$ & $\begin{array}{l}\text { Os } 45 \text { pacientes tinham uma idade média de } 68 \text { anos, todos } \\
\text { portadores de doença arterial periférica, havendo recebido } \\
50 \% \text { deles um número de } 20 \text { sessões, com una pressão de } \\
1.5 \text { atmosferas em } 50 \% \text { dos casos e conseguindo-se uma } \\
\text { satisfatória evolução de seus compromissos em } 77.7 \% \text { dos } \\
\text { casos ( } n=35 \text {, erro padrão=0.03). }\end{array}$ \\
\hline Egito JGTD, et al. & 2013 & $\begin{array}{l}\text { Avaliação da utilização de } \\
\text { oxigenoterapia hiperbárica, como } \\
\text { tratamento adjuvante, em casos de } \\
\text { mediastinite, em pós-operatório de } \\
\text { cirurgia de revascularização } \\
\text { miocárdica. }\end{array}$ & $\begin{array}{l}\text { Identificaram-se } 18 \text { pacientes com mediastinite, nos quais } 33 \\
\text { microrganismos foram isolados, estando a infecção } \\
\text { polimicrobiana presente em } 11 \text { casos. Enterobactérias foram } \\
\text { os germes mais prevalentes e seis agentes multirresistentes. } \\
\text { Ocorreu } 1 \text { óbito, na evolução, } 7 \text { meses após o término da } \\
\text { oxigenoterapia, por septicemia, não relacionado à terapêutica. } \\
\text { O tratamento foi bem tolerado. }\end{array}$ \\
\hline Everett E, Mathioudakis N. & 2018 & $\begin{array}{l}\text { Revisar a justificativa e as diretrizes } \\
\text { para as práticas atuais de cuidado com } \\
\text { as úlceras de pés diabéticos, assim } \\
\text { como sua terapia adjuvante. }\end{array}$ & $\begin{array}{l}\text { A oxigenoterapia hiperbárica foi considerada benéfica na } \\
\text { melhoria das taxas de cicatrização de feridas, embora uma } \\
\text { grande proporção dos dados sejam pequenos ensaios clínicos } \\
\text { randomizados com alto risco de viés. }\end{array}$ \\
\hline Fonsen KM, Thom SR. & 2014 & $\begin{array}{l}\text { Identificar os impactos da hiperóxia nas } \\
\text { células vasculares e nos elementos de } \\
\text { cura da ferida. }\end{array}$ & $\begin{array}{l}\text { O momento ideal para intervenção com oxigenoterapia } \\
\text { hiperbárica em relação a formas mais padrão de terapia, bem } \\
\text { como os desfechos mais apropriados a serem usados para } \\
\text { avaliar os resultados, permanece indefinido. }\end{array}$ \\
\hline Keskin K, et al. & 2017 & $\begin{array}{l}\text { Medir o nível sérico de lipoproteína de } \\
\text { baixa densidade oxidada (LDL-ox) em } \\
\text { pacientes submetidos à oxigenoterapia } \\
\text { hiperbárica. }\end{array}$ & $\begin{array}{l}\text { Não houve diferença significativa entre o nível de LDL-ox dos } \\
\text { pacientes antes e após } \mathrm{OHB}(4,96 \pm 0,1 \text { vs. } 4,94 \pm 0,1 \mathrm{U} / \mathrm{mL} \text {; } \\
\mathrm{p}=0,36) \text {. }\end{array}$ \\
\hline
\end{tabular}

REAS/EJCH | Vol.12(11) | e5282 | DOI: https://doi.org/10.25248/reas.e5282.2020 Página 6 de 11 


\section{Revista Eletrônica Acervo Saúde / Electronic Journal Collection Health | ISSN 2178-2091}

\begin{tabular}{|c|c|c|c|}
\hline Autor & Ano de publicação & Objetivos & Resultados \\
\hline Kimmel HM, et al. & 2016 & $\begin{array}{l}\text { Avaliar a eficácia do uso de oxigênio no } \\
\text { tratamento de feridas complexas. }\end{array}$ & $\begin{array}{l}\text { A oxigenoterapia hiperbárica não ofereceu nenhuma } \\
\text { vantagem adicional no tratamento de feridas, nem apoiou uma } \\
\text { redução nas amputações dos membros inferiores ou no } \\
\text { tamanho da ferida em pacientes com úlceras de pé diabético } \\
\text { durante um período de } 12 \text { semanas. }\end{array}$ \\
\hline Liandro CL, et al. & 2020 & $\begin{array}{l}\text { Descrever a prevalência dos tipos de } \\
\text { feridas com indicação para } \\
\text { oxigenoterapia hiperbárica. }\end{array}$ & $\begin{array}{l}\text { Nos } 60 \text { prontuários analisados foram prevalentes idade entre } \\
19-49 \text { anos e } 61-72 \text { anos (ambas com } 27,1 \%) \text {, sexo masculino } \\
(68,3 \%) \text {, Diabetes Mellitus }(44,1 \% \text { ) e deiscência cirúrgica } \\
(20,3 \%) \text {. Pessoas com idade de } 61-72 \text { anos tiveram maior } \\
\text { tempo de sessões (mediana }=40 \text { sessões). A regressão por } \\
\text { modelagem linear utilizando como preditor a variável tempo de } \\
\text { tratamento pela doença, demonstrou significância estatística } \\
\text { do tabagismo e doença hematológica. }\end{array}$ \\
\hline Rech FV, et al. & 2015 & $\begin{array}{l}\text { Avaliar a morfologia, a área necrótica e } \\
\text { o conteúdo de colágeno em retalhos } \\
\text { cutâneos de ratos submetidos à } \\
\text { oxigenação hiperbárica (OHB). }\end{array}$ & $\begin{array}{l}\text { Os dados mostraram maior área de necrose e menores níveis } \\
\text { de colágeno nas três regiões do grupo de enxerto de pele, } \\
\text { enquanto no grupo enxerto de pele }+ \text { oxigenoterapia } \\
\text { hiperbárica o conteúdo de colágeno foi semelhante aos grupos } \\
\text { que não receberam enxerto de pele. }\end{array}$ \\
\hline Rüttermann M, et al. & 2013 & $\begin{array}{l}\text { Avaliar o tratamento em feridas } \\
\text { crônicas locais em pacientes com } \\
\text { doença vascular periférica, } \\
\text { insuficiência crônica venosa e diabetes. }\end{array}$ & $\begin{array}{l}\text { Esta diretriz contém sete recomendações baseadas em } \\
\text { evidências e } 30 \text { recomendações de boas práticas clínicas } \\
\text { (GCP). Recomendações baseadas em evidências são dadas } \\
\text { a favor do hidrogel, oxigenação hiperbárica e cuidado } \\
\text { integrado, e contra o uso de mel medicinal e fatores de } \\
\text { crescimento. Os termos são definidos precisamente para } \\
\text { facilitar a comunicação e especificar o que se entende por } \\
\text { "desbridamento da ferida" (um procedimento realizado por um } \\
\text { médico) em oposição à limpeza de uma ferida. Sob a premissa } \\
\text { de prevenir a dor, exsudação e maceração, os agentes } \\
\text { terapêuticos locais podem ser escolhidos com base na } \\
\text { evidência científica, na preferência do paciente, na } \\
\text { experiência do médico e na situação da ferida. Os custos } \\
\text { também devem ser considerados. }\end{array}$ \\
\hline
\end{tabular}

Fonte: Menezes EO, et al., 2020.

REAS/EJCH | Vol.12(11) | e5282 | DOI: https://doi.org/10.25248/reas.e5282.2020 Página 7 de 11 


\section{DISCUSSÃO}

Uma ferida crônica é definida como uma área onde a pele não está intacta e que não cicatriza em oito semanas (RÜTTERMANN M, et al., 2013). Essas feridas geralmente se desenvolvem nos membros inferiores como uma complicação de diabetes, insuficiência venosa ou perfusão arterial inadequada. $A$ isquemia vascular crônica com ameaça de membro, por exemplo, é uma síndrome clínica definida pela presença de doença arterial periférica (DAP) em combinação com dor no repouso, gangrena ou ulceração de membro inferior com duração maior que 2 semanas.

As manifestações dessa síndrome, principalmente a de feridas complexas, podem ser beneficiadas da oxigenoterapia hiperbárica, já que a terapia com oxigênio hiperbárico (HBO) promove a cicatrização de feridas em pacientes com doença isquêmica. A síntese de colágeno, a angiogênese e a epitelização, etapas da cicatrização, são extremamente dependentes dos níveis pressóricos de oxigênio. Em ambientes com baixas taxas de oxigênio, tanto a atividade celular quanto a humoral encontram-se prejudicadas, determinando uma incapacidade de manutenção da fisiologia da cicatrização (COSTA RV, et al., 2017).

A oxigenoterapia hiperbárica $(\mathrm{OHB})$ consiste na inalação intermitente de oxigênio $\left(\mathrm{O}_{2}\right)$ a $100 \%$ sob uma pressão atmosférica maior do que 1 atmosfera. $\mathrm{O}$ aumento da pressão de $\mathrm{O}_{2}$ geralmente é expresso em valores múltiplos da pressão atmosférica absoluta (ATA), sendo 1 ATA igual a $1 \mathrm{~kg} / \mathrm{cm}^{2}$ ou $735,5 \mathrm{mmHg}$. De acordo com os protocolos hoje aceitos, a maioria das oxigenoterapias é realizada utilizando-se pressão entre 2 e 3 ATA, exceto para o tratamento da embolia gasosa e da doença descompressiva dos mergulhadores, em que a pressão mais elevada é fator crucial para a obtenção do efeito desejável.

A inalação de ar com elevada pressão parcial de oxigênio proporciona aumento significativo nos níveis de $\mathrm{O}_{2}$ dissolvido no plasma sanguíneo, mas é importante ressaltar que o oxigênio em condições normais de pressão atmosférica não é capaz de produzir as reações observadas na modalidade hiperbárica. Portanto, a câmara compressiva é um instrumento indispensável para sua realização (COSTA RV, et al., 2017).

Durante o tratamento com oxigenoterapia hiperbárica, a tensão arterial de $\mathrm{O}_{2}$ normalmente excede 2.000 $\mathrm{mmHg}$, e níveis de 200-400 mmHg ocorrem nos tecidos (DÜNNWALD T, et al., 2018). Sabe-se que um aumento na produção de espécies reativas de oxigênio ocorre durante o estado de hiperóxia. Embora a toxicidade do oxigênio seja um risco, os protocolos clínicos têm mantido a incidência de efeitos adversos muito baixa, já que as espécies reativas de oxigênio têm efeitos de estresse bioquímicos reversíveis pelas defesas antioxidantes. Segundo Keskin K, et al. (2017), proteínas oxidadas, como a lipoproteína de baixa densidade oxidada (oxLDL), mantém seu nível sérico em pacientes submetidos a, no mínimo, 10 sessões de oxigenoterapia hiperbárica, o que pode evidenciar a segurança do método.

A cicatrização de feridas após uma lesão é um processo complexo que envolve a coordenação de vários mediadores. Logo após a lesão do tecido, o tecido desvitalizado é removido, os queratinócitos migram e proliferam para a borda da ferida e o tecido de granulação, que é composto principalmente de fibroblastos e células endoteliais, começa a se formar. O tecido de granulação contém proliferação neovascular excessiva. Estudos afirmam que o oxigênio deve ser rigidamente controlado em todas as fases da cicatrização de feridas para produzir um tecido de granulação viável, afinal, a hiperóxia tem efeitos em uma série de eventos de sinalização celular que convergem para influenciar o recrutamento, a quimiotaxia celular e a síntese de proteínas que medeiam a cicatrização de feridas (FOSEN, KM et al., 2014).

Segundo Kimmel HM, et al. (2016), o oxigênio está envolvido na hidroxilação de prolina e lisina em prócolágeno, o que leva à maturação do colágeno. Além disso, na angiogênese, a hipóxia é necessária para iniciar o processo de cicatrização de feridas, mas foi demonstrado que, se o oxigênio for administrado, pode acelerar e manter o crescimento dos vasos. A oxigenoterapia hiperbárica, ainda, aumenta a síntese de muitos fatores de crescimento como o VEGF (fator de crescimento endotelial vascular) e TFG (fator de transformação do crescimento), além de regular positivamente o receptor de PDGF (fator de crescimento derivado de plaquetas) em feridas experimentais (FOSEN KM, et al., 2014).

Em feridas cujo tratamento se dá por corticoterapia sistêmica, como o pioderma gangrenoso, a oxigenoterapia hiperbárica mostrou-se segura e eficaz, com poucos efeitos adversos. Segundo Araújo FM, et al. (2013), seu uso como adjuvante se justifica pela redução da dor e infecção, além da aceleração do processo de angiogênese capilar. 
As terapias adjuvantes para feridas complexas, segundo Everett E, et al. (2018), incluem as seguintes categorias: agentes de desbridamento não cirúrgico, curativos e agentes tópicos, oxigenoterapia hiperbárica, terapia de pressão negativa, bioprodutos acelulares, fatores de crescimento humano, terapias baseadas em energia e terapias sistêmicas. No entanto, recomendações baseadas em evidências científicas são encontradas apenas para a oxigenoterapia hiperbárica, práticas de cuidado integrado e contra o uso de mel medicina e fatores de crescimento.

Outros estudos apontam que a oxigenoterapia hiperbárica combinada ao aquecimento de corpo inteiro usando a tecnologia infravermelha de baixa temperatura (LIT) podem ter efeitos sinérgicos positivos no tratamento de lesões por hipóxia. No entanto, Dünnwald T, et al. (2018), demonstrou que é preciso uma sobrecarga de pressão para utilizarmos essa associação, o que pode gerar alterações cardiovasculares moderadas e um aumento na temperatura corporal. Assim, estudos futuros se fazem necessários para comprovar a segurança dessa associação.

Conforme Andrade SM, et al. (2016), a doença arterial periférica, por ser uma doença insidiosa, ocasiona, em muitos casos, má cicatrização de feridas, sobretudo na população idosa. Esses pacientes podem ter feridas com cicatrização prejudicada, em especial quando comparadas às feridas de pacientes jovens, em razão de dieta inadequada, desidratação, descontrole hormonal e imunossupressão. A presença de doenças vasculares, como diabetes e doença arterial periférica, aumenta a prevalência de feridas em idosos, além de resultar no aumento da complexidade dessas feridas.

Segundo Andrade SM, et al. (2016), as feridas que possuem indicação mais frequente para terapia por oxigênio hiperbárico são a úlcera venosa, a lesão traumática aguda e o pé diabético. Nesse estudo, que avaliou 200 pacientes, a maioria era do sexo masculino (51,5\%) e com idade entre $50-73$ anos, concordando com outros estudos de mesma temática. Dentre os fatores de risco, 189 pacientes negaram o tabagismo e 58 possuíam doenças cardiovasculares. É importante mencionar que, no que diz respeito ao tabagismo, destoando do fato de que este é um fator de risco para as lesões por doença arterial periférica, a maioria dos pacientes do estudo não possuía tal hábito. Quando comparados aos pacientes com feridas agudas, os portadores de feridas crônicas precisaram de menos sessões e conseguiram ter suas feridas reduzidas ou cicatrizadas.

Um dos ensaios com maior número de pacientes é o estudo HODFU (Terapia de Oxigênio Hiperbárico em Diabéticos com Úlceras Crônicas do Pé). Neste, foram inscritos 96 pacientes que foram distribuídos aleatoriamente em 2 grupos, um dos quais recebeu oxigênio hiperbárico e o outro ar hiperbárico, seguindo um protocolo duplo-cego. Sessões de 90 minutos foram realizadas diariamente, 5 dias por semana, durante 8 semanas. Pacientes com vários graus de úlceras diabéticas com mais de 3 meses de idade foram incluídos. Dos pacientes que receberam oxigênio hiperbárico, $52 \%$ curaram suas feridas, enquanto $27 \%$ do grupo controle cicatrizaram. Houve 3 amputações no grupo intervencionado e 2 amputações no grupo placebo (BERNER JE, et al., 2014).

Em um estudo com 45 pacientes com úlcera por doença arterial periférica feito no Hospital Militar Central de Bogotá, com $53 \%$ dos pacientes do sexo masculino, a média de sessões de oxigenoterapia hiperbárica, para tratamento de úlceras arteriais que predominavam exclusivamente em membros inferiores, na maioria dos casos relacionados à doença diabética, foi de 26 sessões. $77,7 \%$ das pessoas da população estudada tiveram resultados satisfatórios, representando o fechamento da úlcera arterial. 15,5\% das pessoas não apresentaram melhora nas úlceras arteriais, permanecendo nas mesmas condições detectadas na avaliação clínica inicial (DURÁN EP, et al., 2012).

Podemos observar que os resultados da oxigenoterapia hiperbárica foram significantes em poucas sessões, em um tempo curto inferior ao tempo que se utiliza em outras formas de tratamento e com resultado superior, sendo, ainda, mínimo os riscos de complicações da terapia.

Já no estudo transversal de Liandro CL, et al. (2020), com 60 pacientes, realizado em um hospital no Rio de Janeiro, a média de sessões foi de 40 para pacientes entre 61-89 anos, enquanto pacientes com 19 a 49 anos realizavam apenas, em média, 20 sessões. Apesar de nesse estudo a maioria da população ser do sexo

REAS/EJCH | Vol.12(11) | e5282 | DOI: https://doi.org/10.25248/reas.e5282.2020 Página 9 de 11 
masculino, seguindo o perfil epidemiológico demonstrado no estudo de Andrade SM, et al. (2016), o tabagismo foi encontrado em $73,3 \%$ dos pacientes desse estudo, pactuando com grande parte da literatura disponível sobre o tema. Em relação prevalência do sexo masculino em todos os artigos revisados, acreditase que que este resultado pode ser explicado pela resistência dos homens em procurar serviços de saúde e, quando procuram, já existe um quadro clínico de lesão crônica instalado.

Em relação a lesões dos cotos de amputações por doença arterial periférica, Columbo JA, et al. (2016), descreve um relato de 4 casos de pacientes com amputações acima do joelho, cujas feridas no coto não cicatrizaram, mesmo após inúmeras tentativas de revascularização. Com a adição do tratamento com oxigênio hiperbárico ao cuidado vigilante da ferida e à terapia de pressão negativa, esses pacientes curaram seus cotos de amputação e receberam próteses, podendo deambular sem dor. Esse estudo demonstrou que a oxigenoterapia hiperbárica, como tratamento adjuvante, pode evitar operações de alta morbidade e mortalidade, melhorando a cicatrização de feridas nesse grupo de pacientes com amputações.

Segundo Rech FV, et al. (2015), alguns efeitos colaterais elucidados da oxigenoterapia hiperbárica são o barotrauma de orelha média, que ocorre em mais de $2 \%$ dos pacientes e pode ser prevenida ou pelo menos minimizada pela inserção de tubos de timpanostomia e claustrofobia, a dispneia pulmonar, tosse e dor a inspiração. No entanto esses efeitos são raros e, apesar deles, acredita-se que essa terapia leve a benefícios hemodinâmicos, como já demonstrado nesta revisão.

\section{CONSIDERAÇÕES FINAIS}

Apesar das limitações metodológicas e da grande proporção dos dados serem de pequenos ensaios clínicos randomizados, os estudos apresentados nessa revisão demonstraram que a oxigenoterapia hiperbárica apresenta boa eficácia no tratamento adjuvante de feridas complexas, incluindo as lesões por doença arterial periférica, úlceras em pés diabéticos e feridas agudas relacionadas a trauma. Além disso, foi possível delinear um perfil epidemiológico dessas lesões, que, nos artigos aqui apresentados, demonstrou serem mais prevalentes no sexo masculino em adultos ou na população idosa. A revisão também evidenciou que as principais doenças relacionadas a essas lesões, além da doença arterial periférica, são o diabetes mellitus, a hipertensão arterial sistêmica e a associação destas com o tabagismo. Dessa forma, por serem doenças extremamente prevalentes em nossa população, faz-se necessário um maior número de estudos prospectivos nessa área, além de um esforço constante para melhoria desses fatores de risco. É importante ressaltar, contudo, a necessidade de empregar a oxigenoterapia hiperbárica de forma responsável e por profissionais qualificados.

\section{REFERÊNCIAS}

1. ADORNO ETF, et al. Perfil epidemiológico dos pacientes tratados com auxílio da oxigenioterapia hiperbárica no estado de Mato Grosso do Sul de maio de 2007 a outubro de 2012. Revista brasileira de cirurgia plástica, 2013. 28(4): 650-654.

2. ANDRADE SBD, SANTOS ICRV. Oxigenoterapia hiperbárica para tratamento de feridas. Revista Gaúcha de Enfermagem, 2016;37(2), e59257.

3. ARAUJO FM, et al. Pioderma gangrenoso: enxerto de pele e oxigenoterapia hiperbárica como adjuvantes no tratamento de úlcera extensa e profunda. Anais Brasileiros de Dermatologia, 2013;88(6, Suppl. 1), 176-178.

4. BENEDICT, C. et al. Atypical Wounds; Hyperbaric Oxygen Therapy. Journal Clinics in Podiatric Medicine and Surgery, Elsevier, 2019; (36).

5. BERNER JE, et al. Uso de oxígeno hiperbárico para el manejo de heridas: bases físicas, biológicas y evidencia disponible. Revista médica de Chile, 2014;142(12), 1575-1583.

6. COLUMBO JA, et al. Hyperbaric oxygen for patients with above-knee amputations, persistent ischemia, and nonreconstructable vascular disease. J Vasc Surg. 2016;63(4):1082-1084.

7. COSTA RV, et al. O papel da oxigenoterapia hiperbárica na doença vascular periférica. Jornal Vascular Brasileiro, São Paulo, 2003; 2(3):177-82.

8. DÜNNWALD T, et al. Combined Hyperbaric Oxygen Partial Pressure at 1.4 Bar with Infrared Radiation: A Useful Tool to Improve Tissue Hypoxemia? Med Sci Monit. 2018; 24:4009-4019.

9. DURAN EP, et al. Resultados de un programa de oxígenoterapia hiprbárica para el tratamiento de úlceras arteriales. Rev. MED. 2012; 20(2): 21-29. 
10. EGITO, JGTD, et al. Evolução clínica de pacientes com mediastinite pós-cirurgia de revascularização miocárdica submetidos à oxigenoterapia hiperbárica como terapia adjuvante. Einstein (São Paulo), 2013;11(3), 345-349.

11. EVERETT E, MATHIOUDAKIS $\mathrm{N}$. Update on management of diabetic foot ulcers. Ann $N$ Y Acad Sci. 2018;1411(1):153-165.

12. FOSEN KM, THOM SR. Hyperbaric oxygen, vasculogenic stem cells, and wound healing. Antioxid Redox Signal. 2014;21(11):1634-1647.

13. KESKIN K, et al. Serum oxidized low-density lipoprotein level as a marker of oxidative stress in patients undergoing hyperbaric oxygen therapy. Turk Kardiyol Dern Ars. 2017;45(6):533-537.

14. KIMMEL HM, et al. The Presence of Oxygen in Wound Healing. Wounds. 2016;28(8):264-270.

15. LIANDRO CL, et al. Oxigenoterapia hiperbárica como tratamento adjuvante para feridas: estudo de prevalência. Enfermagem em foco. Brasília, 2020; 11(2): 29-34.

16. LIN P. et al. Hyperbaric Oxygen Therapy Enhanced Circulating Levels of Endothelial Progenitor Cells and Angiogenesis Biomarkers, Blood Flow, in Ischemic Areas in Patients with Peripheral Arterial Occlusive Disease. Journal of Clinical Medicine, Royall College of Physicians, Londres, 2018; (14).

17. MARTINELLI B, et al. Alterações cardiorrespiratórias de pacientes submetidos à oxigenoterapia hiperbárica. Revista da escola de enfermagem da USP, São Paulo, 2019;(53).

18. NAKAMURA $\mathrm{H}$, et al. Changes in Skin Perfusion Pressure After Hyperbaric Oxygen Therapy Following Revascularization in Patients with Critical Limb Ischemia: A Preliminary Study. The International Journal of Lower Extremity Wounds, SAGE Publications, 2020;(62).

19. RECH FV, et al. Action of hyperbaric oxygenation in the rat skin flap. Acta Cirurgica Brasileira, 2015;30(4), 235-241.

20. RÜTTERMANN M, et al. Local treatment of chronic wounds: in patients with peripheral vascular disease, chronic venous insufficiency, and diabetes. Dtsch Arztebl Int. 2013;110(3):25-31.

21. SILVA D, et al. Prescrição de exercícios físicos para pessoas com doença vascular periférica. Revista Brasileira de Ciência e Movimento, Brasília, 2002; 1(10).

22. TUMA JR. P, et al. Effect of hyperbaric oxygen on the regeneration of experimental crush injuries of nerves. Revista do Hospital das Clínicas, 1999;54(3), 81-84. 